\title{
Experiencias sobre la utilización de gas natural en la fabricación de clínker
}

\author{
SANTIACO GIBERT FORTET \\ JORGE COROMINA CASADELLA \\ Ingenieros Químicos I.Q.S. \\ Auxiliar de la Construcción, S. A.
}

\section{INTRODUCCION}

Cuando en 1967 GAS NATURAL, S. A. de Barcelona realizaba los estudios del mercado potencial de consumidores de gas natural, en el área de Barcelona, se establecieron conversaciones sobre la posibilidad de emplearlo en la fábrica de Cementos Sanson, que L.A.C.S.A. posee en Sant Felíu de Llobregat.

A consecuencia de las mismas, se estudiaron con detalle:

1. Las inversiones en:

Red hasta fábrica.

Red interior.

Celdas de regulación y medidas.

Celdas de protección.

Mechero, etc.

2. Los riesgos que significaba efectuar la producción, en cantidad y/o calidad, durante las pruebas o período de adaptación de un nuevo combustible.

3. Las ventajas de disponer de un combustible sustituto del fuel-oil, dado que el mercado del carbón en España descartaba a éste para su empleo en los hornos de clínker.

4. ${ }^{\circ}$ La disminución de la contaminación, no sólo por la reducción del contenido de $\mathrm{SO}_{2}$ en humos, sino por mejorar los rendimientos de los filtros electrostáticos al aumentar el contenido de humedad en aquéllos.

Desde el punto de vista de posibles aumentos o disminución de rendimientos energéticos o capacidad de producción, los datos disponibles no permilitieron prejuzgar en que sentido se efectuarían.

Por el hecho de haber sido La Auxiliar de la Construcción en la factoría de San Justo Desvern la pionera del empleo del fuel-oil en España para la fabricación de clínker, también existía el interés de ser los pioneros del gas natural.

A consecuencia de todo ello, durante el 68 se estableció un plan de trabajo conjunto con GAS NATURAL, tanto desde el punto de vista técnico como económico, que permitió establecer un protocolo de contrato, de forma que en la primavera del 69 se empezaron las pruebas de consumo de gas natural en el primer horno y, paulatinamente, su incorporación como combustible normal. 


\section{GENERALIDADES SOBRE EL GAS NATURAL}

\subsection{Datos sobre el gas natural en el mundo y su empleo en cementos}

Con el fin de situar al Gas Natural dentro del balance mundial de energía disponible, analicemos brevemente, y en primer lugar, los consumos y reservas mundiales para detenernos después en su utilización en la Industria del Cemento.

\subsubsection{Reparto mundial de energía consumida (1)}

Del total de la energía consumida en el mundo en el año 1973 un $56 \%$ fue de petróleo, mientras que el gas natural y el carbón quedaron nivelados en un $18 \%$ (tabla 1).

Estimaciones aproximadas señalan que para el año 1990 el consumo energético en carbón, gas natural y nuclear serán equivalentes, situándose entre un 15 y un $20 \%$ del total.

TABLA 1

\begin{tabular}{|c|c|c|c|c|}
\hline & \multicolumn{2}{|c|}{1973} & \multicolumn{2}{|c|}{1990} \\
\hline & $\begin{array}{l}\text { MM b/d equival. } \\
\left(\times 166=\mathrm{Mm}^{3} / \mathrm{d}\right)\end{array}$ & $\%$ & $\begin{array}{l}\text { MM b/d } \\
\text { equivalentes }\end{array}$ & $\%$ \\
\hline $\begin{array}{l}\text { Carbón } \\
\text { Petróleo } \\
\text { Gas natural } \\
\text { Hidráulica } \\
\text { Nuclear } \\
\text { Otros }\end{array}$ & $\begin{array}{r}16 \\
48 \\
16 \\
6 \\
1 \\
-\end{array}$ & $\begin{array}{r}18 \\
56 \\
18 \\
7 \\
1 \\
\end{array}$ & $\begin{array}{c}25-40 \\
63-71 \\
20-23 \\
10-12 \\
25-30 \\
2-4\end{array}$ & $\begin{array}{c}17-22 \\
44-39 \\
14-13 \\
7-7 \\
17-17 \\
1-2\end{array}$ \\
\hline
\end{tabular}

\subsubsection{Reservas de gas y distribución de consumos}

El total de reservas de gas natural en el mundo en el año 1974 se estimaron en $64 \times 10^{12}$ $\mathrm{m}^{3}$ (1). La mayor reserva mundial se atribuía a la URSS con un $36 \%$, seguido por Oriente Medio (24\%) y Norte América (14\%) (tabla 2).

TAB L A 2

\begin{tabular}{|ll|}
\hline Total reservas en $1974: 64 \times 10^{12} \mathbf{m}^{3}(\mathbf{1})$ \\
\hline $36 \%$ & URSS \\
$24 \%$ & Oriente Medio \\
$14 \%$ & Norte América \\
$9 \%$ & Europa Occidental \\
$8 \%$ & Africa \\
$5 \%$ & Extremo Oriente \\
$3 \%$ & Sudamérica \\
$1 \%$ & Europa Oriental + China $\ldots$ \\
\hline
\end{tabular}


El consumo de gas natural estimado en 1974 fue de $1,21 \times 10^{12} \mathrm{~m}^{3}$ (1), correspondiendo más de la mitad a Norte América, seguido de la URSS con un $19 \%$ (fig. 1).

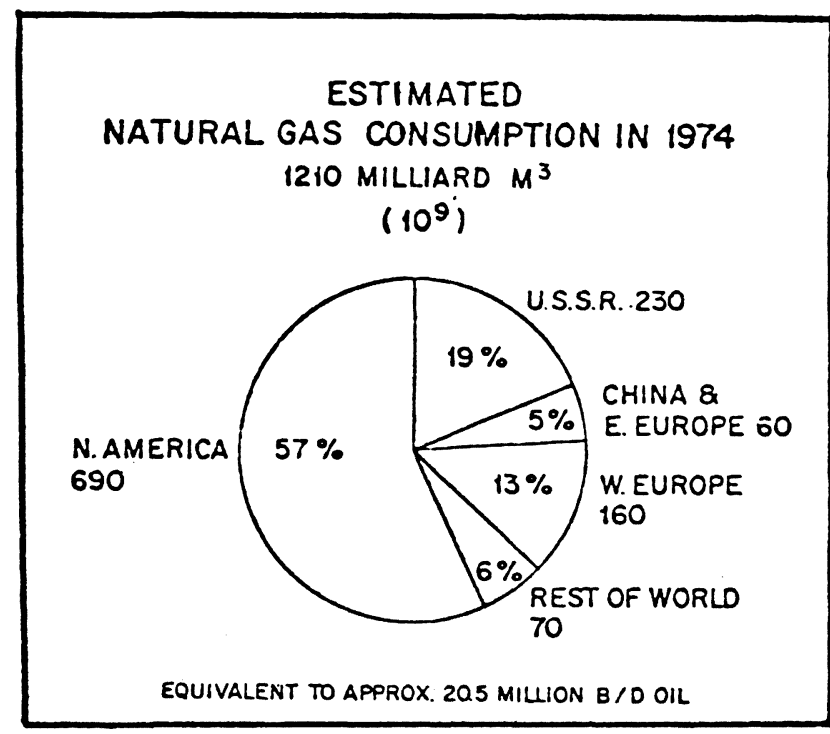

Fig. 1

\subsubsection{Consumo de gas en la fabricación de cemento}

La industria del cemento consume en general proporciones pequeñas de gas natural, respecto al consumo del resto de industrias. Tenemos, por ejemplo, los casos de Francia y G. Bretaña que en 1973 consumieron en cemento el 4,5\% del gas natural consumido en la industria (tabla 3).

TAB L A 3

\begin{tabular}{|l|c|c|c|}
\hline \multicolumn{1}{|c|}{ Año 1973 } & $\begin{array}{c}\text { Consumo total } \\
\text { Tcal (3) }\end{array}$ & \% Consumo Ind. & $\begin{array}{c}\text { \% } \\
\text { Cons. cemento } \\
\text { respecto ind. } \\
\text { (4) }\end{array}$ \\
\hline & & & \\
Austria & 34.460 & 80,8 & \\
Bélgica & 77.846 & 79,2 & \\
Canadá & 257.398 & 54,3 & \\
Checoslovaquia & 31.862 & 66,3 & 4,5 \\
Rep. Fed. Alemana & 352.516 & 75,9 & 3,3 \\
Francia & 145.353 & 58,8 & 1,2 \\
Rep. Dem. Alemana & 37.269 & 79,3 & 4,5 \\
Italia & 154.744 & 71,2 & $(2,3)$ \\
Holanda & 315.106 & 60,7 & 1,9 \\
Rumania & 165.706 & 83,6 & \\
G. Bretaña & 271.656 & 45,3 & \\
USA & 5.066 .133 & 61,4 & \\
URSS & 1.827 .121 & 85,7 & \\
España & 7.656 & 74,4 & \\
& & & \\
\hline
\end{tabular}




\subsubsection{Evolución del empleo de combustibles en la industria del cemento}

Centrándonos en la industria del cemento, el combustible utilizado mayoritariamente en Europa es el fuel-oil. De los datos recopilados del año 1974, el país que mayor proporción de gas natural consumió en la industria del cemento fue Alemania Occidental, con unas proporciones de $72 \%$ de fuel, $21 \%$ de gas natural y $7 \%$ de carbón (tabla 4).

\section{T A B L A 4}

\begin{tabular}{|c|c|c|c|c|c|c|c|c|c|}
\hline & \multicolumn{3}{|c|}{1962 (2) } & \multicolumn{3}{|c|}{1973 (4) (5) } & \multicolumn{3}{|c|}{$1974(5)$} \\
\hline & c & F.o & G.N & c & F.O & G.N & c & F.O & G.N \\
\hline $\begin{array}{l}\text { Alemania Or. } \\
\text { Francia } \\
\text { Italia } \\
\text { Luxemburgo } \\
\text { URSS } \\
\text { G. Bretaña } \\
\text { USA } \\
\text { Alemania Osc. }\end{array}$ & $\begin{array}{l}99,0 \\
80,4 \\
18,0 \\
79,5 \\
37,7 \\
63,5 \\
47,6 \\
68,5\end{array}$ & $\begin{array}{c}0 \\
14,1 \\
68,0 \\
0 \\
10,5 \\
36,5 \\
6,0 \\
31,0\end{array}$ & $\begin{array}{r}1,0 \\
5,1 \\
14,0 \\
20,5 \\
51,8 \\
0 \\
46,4 \\
0,5\end{array}$ & $\begin{array}{l}4,0 \\
1,5\end{array}$ & $\begin{array}{l}85,0 \\
85,8\end{array}$ & $\begin{array}{l}11,0 \\
12,7 \\
14,3 \\
29,0\end{array}$ & $\begin{array}{l}4 \\
1\end{array}$ & $\begin{array}{l}85,0 \\
84,7\end{array}$ & $\begin{array}{l}11,0 \\
13,6\end{array}$ \\
\hline
\end{tabular}

\subsubsection{Desarrollo del gas natural en España}

España inició la importación de gas natural licuado en el año 1969, para abastecer el área de Barcelona. El consumo en cemento se inició ya desde el primer momento, si bien su consumo fue en principio reducido.

En el pasado año 1975 el consumo total de gas natural fue de 8.560 Tcal que equivalen a $8,5 \times 10^{8} \mathrm{~m}^{3}$, de las que la industria cementera empleó un $3,18 \%$. La totalidad del consumo de la industria cementera corresponde a la planta de la Auxiliar de la Construcción, S. A. de San Felíu de Llobregat (Cementos Sanson).

El reparto de combustible utilizado en dicha planta en 1975 fue de un $58 \%$ de fuel-oil y un $42 \%$ de gas natural.

\subsection{Combustibles líquidos y gaseosos}

A continuación describimos, de modo resumido, las principales características de ambos tipos de combustible usados en la industria cementera.

También se analizan, brevemente, las influencias observadas en su utilización debidas a su distinta naturaleza.

\subsubsection{Principales caracteristicas}

El gas natural empleado en Barcelona está básicamente compuesto por una mezcla de $84 \%$ de metano y un $16 \%$ de etano. Además posee también trazas de propano, butano y nitrógeno. 
En la tabla 5 aparece la composición volumétrica, así como sus principales características de combustión.

TAB L A 5

Características del Gas Natural de Barcelona

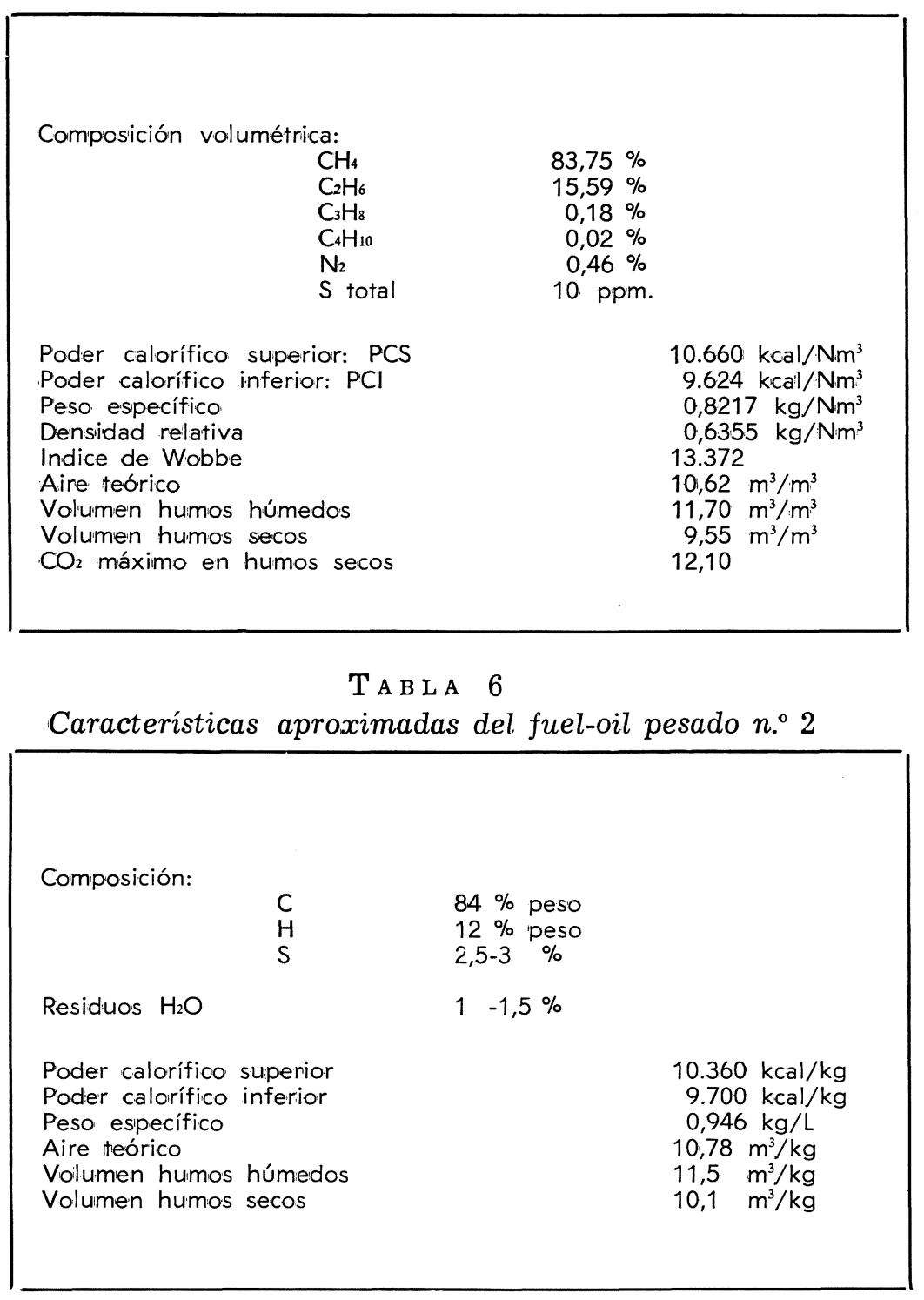

Las características del fuel-oil aparecen en la tabla 6. Comparando ambos combustibles se observa que el contenido calórico del $\mathrm{kg}$ de fuel-oil se asemeja al del $\mathrm{Nm}^{3}$ de gas natural. Por otra parte, se deduce que las necesidades de aire por calor útil se diferencian en un $0,6 \%$, es decir, el gas natural requiere un $0,6 \%$ menos de aire que el fuel.

2.2.2. Consideraciones sobre la utilización del fuel-oil y gas natural para la fabricación de clinker

Dada la distinta naturaleza de ambos combustibles, es factible exponer las siguientes consideraciones:

a) La mezcla de combustible-carburante puede ajustarse mejor mediante un combustible gaseoso, dado que su dispersión a escala molecular facilita esta mezcla frente a la pulverización de fuel. 
b) El encendido es más cómodo que en el caso del fuel-oil.

c) La mayor claridad en la atmósfera del horno utilizando gas natural permite una inspección ocular más completa, especialmente en el caso de enfriador de parrilla. Esto seguramente implica una mayor efectividad de los sistemas de control de radiacción o cámaras de televisión.

d) Las inversiones necesarias para una instalación de gas natural, suponiendo el gas a pie de fábrica, así como los gastos de mantenimiento y de uso, son inferiores que los propios del fuel y todavía más respecto al carbón (10).

e) El volumen de gases de combustión del gas natural es del orden de un 3-4 \% superior al fuel-oil, lo cual en algunos casos puede limitar la producción del horno.

En la bibliografía y las publicaciones más recientes se encuentran muchas contradicciones en cuanto a los rendimientos obtenidos con el gas natural y el fuel-oil. Datos recientes de la URSS (3) cifran un ahorro en calorías del $6 \%$ respecto al carbón, y en Alemania Oriental (10) ahorros del $4,2 \%$ respecto al fuel.

Sin embargo, según otras informaciones (11), los resultados obtenidos son de un $5 \%$ superiores respecto al fuel-oil y en algunos, incluso, se han visto obligados a disminuir producciones.

Es difícil generalizar sobre las ventajas de uno $u$ otro combustible. Parece ser que tiene gran importancia el tipo de quemador empleado: hay casos en que en el mismo horno se han obtenido resultados totalmente opuestos usando diferentes quemadores (10).

Seguramente debido a la versatilidad de la llama de gas natural, hace que la influencia del quemador sea importantísima, lo cual puede explicar las contradicciones que hay en la bibliografía actual sobre el tema.

\section{EQUIPOS DE COMBUSTION}

Los equipos de combustión que se han empleado para el gas natural en la industria del cemento han ido evolucionando sustancialmente. Podemos citar los principales, así como las tendencias actuales sobre la construcción de quemadores.

En un principio se empleaba un simple tubo, que en algunos casos se ramificaba en sus extremos produciendo varias salidas paralelas de gas.

Posteriormente, se han empleado quemadores con una salida única a través de un inyector calibrado y circundado por aire primario en rotación; éstos son los quemadores llamados de jet simple.

Actualmente los quemadores suelen disponer de varios flujos de gas a diferentes presiones. Con ello se consigue una mayor capacidad para variar la llama y acondicionarla a los diferentes tipos de hornos. De este tipo de quemadores, los más usuales son con dos flujos de gas (jet doble): uno central según el eje del horno y otro anular cuya salida puede variar sustancialmente, desde salidas completamente radiales que se mezclan con el aire primario, hasta salidas inclinadas, tangenciales y otras variantes.

Esta última técnica de quemadores ha sido muy desarrollada en Francia por el "Centre d'Etudes des Flames du Gaz Naturel", obteniendo buenos resultados y llegando a eliminar el aire primario de la combustión. 
Por otra parte, en Alemania Oriental y la URSS (10) se ha desarrollado y perfeccionado últimamente el quemador de jet simple, obteniendo, al parecer, mejores resultados que con quemadores de jet doble.

En la combustión de gas natural la cantidad de aire primario empleado es inferior al $10 \%$. Actualmente, como hemos citado, incluso se ha suprimido.

En general, tanto si se trata de quemadores de jet simple o dobles, la posibilidad de cambio de combustible es rápida e, incluso, algunos modelos pueden consumir simultáneamente combustibles líquidos y gaseosos.

Visto de forma general los principales tipos de quemadores, explicaremos a continuación los equipos empleados en L.A.C.S.A.

\subsection{Equipos empleados en L.A.C.S.A.}

El gas natural se suministra desde el puerto de Barcelona, llegando a la fábrica a una presión entre 5 y $10 \mathrm{kp} / \mathrm{cm}^{2}$; después de filtrado se reduce la presión a $2 \mathrm{kp} / \mathrm{cm}^{2}$, distribuyéndose a los hornos a esta presión.

El sistema de seguridad de la instalación de recepción consiste en cortar automáticamente el paso de gas a los hornos en caso de falta de presión en la tubería de baja presión.

También dispone de una válvula de seguridad para el caso de un exceso de presión en la tubería de baja.

El sistema de seguridad en los hornos consiste en cortar el paso de gas en caso de falta de aire primario en el quemador, corte de corriente o paro del ventilador del horno.

En cuanto a quemadores hemos ensayado cuatro tipos distintos.

En un principio se utilizó un mechero tipo Del Monego s.p.a. (fig. 2) de diseño sencillo, que consiste en un único jet de gas rodeado por aire primario. La regulación se conseguía mediante el movimiento del inyector central a través de una salida en forma de venturi.

Posteriormente se utilizó un quemador Unitherm, mixto para gas-fuel, consistente en una salida central de fuel rodeada por aire primario al que se mezclaba el gas por una batería de tubos concéntricos (fig. 3). La regulación consistía en provocar una mezcla más o menos intensa de gas-aire.

Estos quemadores fueron sustituidos por otros dos tipos Vasaco-Coen, que son los empleados actualmente.

En uno de los hornos se utiliza un mechero de diseño complicado, pero que ha demostrado ser muy versátil en cuanto a posibilidad de variación de la llama y de mezcla de combustibles gas-fuel. Dispone de tres conducciones de gas concéntricas, pudiendo sustituirse la central por fuel. Las dos salidas centrales son paralelas al eje del horno, mientras que la salida más exterior es en dirección radial (fig. 4). Variando los caudales de las tres conducciones se obtienen las más diversas formas de llama.

El otro tipo de mechero tipo Vasaco-Coen, actualmente en servicio, es el clásico quemador de doble jet con una salida central de gas y otra radial rodeadas por aire primario (fig. 5). Este mechero se encuentra en servicio desde febrero del 75 con excelentes resultados. 
Todos estos mecheros presentan, como característica común, unas aletas en la conducción de aire primario que permiten provocar una mayor o menor turbulencia en la salida.

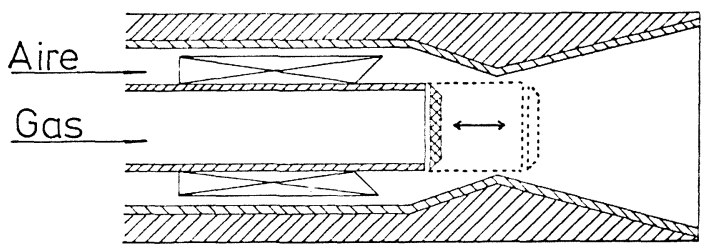

Fig. 2

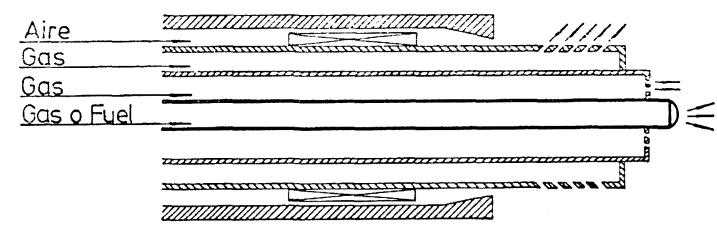

Fig. 4

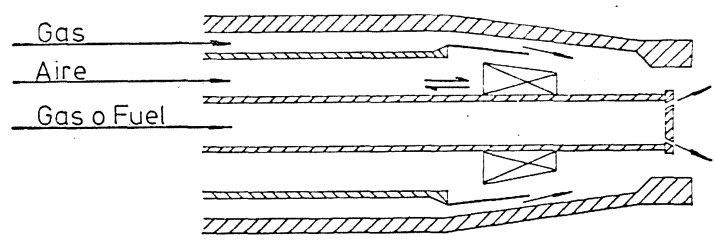

Fig. 3

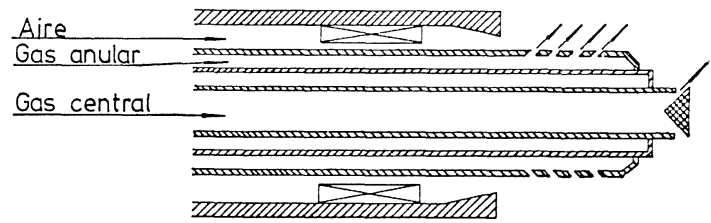

Fig. 5

\section{INFLUencia DeL GAS EN EL PROCESO}

Trataremos a continuación de una serie de problemas de tipo químico que se nos presentaron en la aplicación del gas natural. Debemos señalar ante todo que los problemas que citamos son aplicables a nuestro caso concreto, en razón de las materias primas que utilizamos, por lo que no pueden ser generalizados a otras plantas.

\subsection{Primeras experiencias}

Nuestras primeras experiencias datan de mayo del 69.

Las primeras pruebas se realizaron en un horno Smidht, vía seca, de 1.100 t/día, con enfriaderos satélites. El quemador utilizado fue el tipo Del Monego.

Los resultados obtenidos fueron bastante deficientes y pudimos observar las siguientes diferencias entre el clínker obtenido en el mismo horno usando fuel y gas natural:

a) Peso aparente del clínker más alto del normalmente obtenido utilizando fuel.

b) Un aumento considerable de cal libre, pasando de valores entre 0,8-1,0 con fuel a valores superiores a 2,5 .

c) Como consecuencia del apartado anterior, el clínker obtenido era expansivo.

d) Notable descenso de las resistencias iniciales del cemento fabricado con este clínker que, sin embargo, se recuperaba a los 28 días con valores normales.

e) Descenso del contenido en $\mathrm{SO}_{3}$ en el clínker, como era de esperar, debido al nulo contenido de $\mathrm{S}$ en el gas natural. 
Los ensayos microscópicos de este clínker mostraban gran descenso del tamaño de cristales de $\mathrm{SC}_{3}$, apareciendo poco definidos, y zonas de $\mathrm{SC}_{2}$ ameboide junto a otras de cal libre.

\subsection{Mezcla de combustibles}

Como consecuencia de estos resultados poco alentadores se decidió probar una mezcla de combustibles gas-fuel.

Este ensayo se realizó a partir de abril del 70 en un horno Humboldt de 4 etapas, 250 t/día de producción, con enfriador de parrilla y utilizando el mechero Unitherm.

El porcentaje de fuel en calorías aportadas era de un $17 \%$. Los resultados obtenidos fueron excelentes; todos los problemas que tuvimos en la primera fase quedaron superados y el clínker obtenido era de una calidad igual o superior al obtenido con fuel.

Hasta el año 1971 se siguió con esta mezcla de combustibles, pero de repente aparecieron de nuevo las características iniciales, o sea negativas, del clínker fabricado con gas natural.

Se aumentó el \% de fuel en la mezcla de combustibles, llegando a un $25 \%$ en calorías aportadas, pero, a pesar de ello, no pudo conseguirse un clínker aceptable.

Llegados a este punto empezamos a preguntarnos cuál era el motivo del cambio experimentado, ya que en principio no había ninguna variación apreciable en el crudo ni en el funcionamiento del horno.

\subsection{Influencia del $\mathrm{SO}_{3}$ y los álcalis}

La confrontación de nuestros datos con las numerosas referencias bibliográficas consultadas, nos llevaron a la conclusión de que el problema estaba en el equilibrio $\mathrm{SO}_{3}$-álcalis.

La influencia del $\mathrm{SO}_{3}$ en el proceso de fabricación del clínker va ligada a los álcalis.

$\mathrm{El} \mathrm{SO}_{3}$ producido en el horno reacciona preferentemente con los álcalis, para formar una solución sólida de sulfatos sódico-potásico, reaccionando con mayor facilidad con el potasio que con el sodio. Los sulfatos alcalinos abandonan el horno debido a su escasa volatilidad en comparación con los otros compuestos alcalinos (cloruros) (6) (8) (9).

Un exceso de $\mathrm{SO}_{3}$ en relación a los álcalis presentes puede reaccionar con los silicatos bicálcico y aluminatos, dando lugar a pegaduras e incluso anillos en el interior del horno.

Un defecto de $\mathrm{SO}_{3}$ en relación a los álcalis presentes deja libres en el interior del horno una mayor cantidad de $\mathrm{K}_{2} \mathrm{O}$ y $\mathrm{Na}_{2} \mathrm{O}$, que son capaces de combinar con otras fases del clínker.

Ha sido confirmada la existencia en el clínker de una fase de aluminato tricálcico estabilizado por $\mathrm{Na}_{2} \mathrm{O}$ que se ajusta a la composición $\mathrm{A}_{3} \mathrm{C}_{8} \mathrm{~N}$ (6) (8). 
$\mathrm{Ha}$ sido igualmente comprobado que el $\mathrm{K}_{2} \mathrm{O}$ puede ser combinado como solución sólida en el $\mathrm{SC}_{2}$ con una composición que se ajusta a $\mathrm{S}_{12} \mathrm{C}_{23} \mathrm{~K}$ y que puede interpretarse como 12 moles de $\mathrm{SC}_{2}$ estabilizados por uno de $\mathrm{K}_{2} \mathrm{O}$ (6) (7) (9).

$$
12 \mathrm{SC}_{2}+\mathrm{K}_{2} \mathrm{O} \rightarrow \mathrm{S}_{12} \mathrm{C}_{23} \mathrm{~K}+\mathrm{CaO} \quad \text { (A) }
$$

Vemos, pues, que la presencia de $\mathrm{K}_{2} \mathrm{O}$ que no ha sido combinado como $\mathrm{SO}_{4} \mathrm{~K}_{2}$ puede dar lugar al $\mathrm{S}_{12} \mathrm{C}_{23} \mathrm{~K}$, que no queda disponible para formar $\mathrm{SC}_{3}$ y con lo que resulta reducida la cantidad de $\mathrm{CaO}$ combinable con los silicatos $\mathrm{y}$, en consecuencia, queda reducido el contenido en $\mathrm{SC}_{3}(6)$.

Para hacernos una i.dea de esta reducción, haciendo números con la anterior reacción, vemos que un $1 \%$ de $\mathrm{K}_{2} \mathrm{O}$ inhabilita a un $22 \%$ de $\mathrm{SC}_{2}$ para dar lugar a la misma cantidad de $\mathrm{S}_{12} \mathrm{C}_{23} \mathrm{~K}$ y liberando un $0,6 \%$ de $\mathrm{CaO}$. Esta cal liberada queda disponible para reaccionar con $\mathrm{SC}_{2}$ para formar un $2,5 \%$ de $\mathrm{SC}_{3}$.

Como balance de todo ello vemos que un $1 \%$ de $\mathrm{K}_{2} \mathrm{O}$ puede llegar a retirar del clínker a un $24 \%$ de $\mathrm{SC}_{2}$ (7).

Todo ello queda resumido en el siguiente esquema:

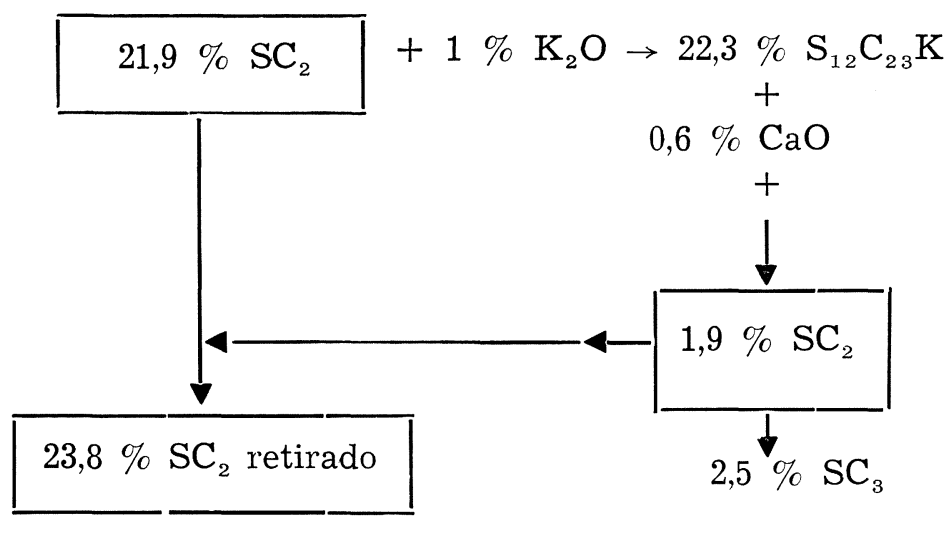

Todavía puede acentuarse más la importancia de los efectos de la combinación del $\mathrm{K}_{2} \mathrm{O}$ con el $\mathrm{SC}_{2}$ si consideramos el caso en que la cantidad de $\mathrm{SC}_{2}$ disponible en el clínker es insuficiente para equilibrar al $\mathrm{K}_{2} \mathrm{O}$. En este caso es factible considerar que se descompone la fase $\mathrm{SC}_{3}$ de acuerdo con:

$$
12 \mathrm{SC}_{3}+\mathrm{K}_{2} \mathrm{O} \rightarrow \mathrm{S}_{12} \mathrm{C}_{23} \mathrm{~K}+13 \mathrm{CaO}
$$

A resultas de ello por cada un $1 \%$ de $\mathrm{K}_{2} \mathrm{O}$ se reduce el $\mathrm{SC}_{3}$ en un $29 \%$, liberándose un $7,7 \%$ de cal libre.

El mecanismo de lo expuesto anteriormente puede ser resumido de la siguiente forma:

a) $\mathrm{El} \mathrm{SO} \mathrm{SO}_{3}$ combina con el $\mathrm{K}_{2} \mathrm{O}$ dando lugar a $\mathrm{SO}_{4} \mathrm{~K}_{2}$.

b) $\mathrm{El} \mathrm{K}_{2} \mathrm{O}$ que aún queda disponible puede combinarse con el $\mathrm{SC}_{2}$, quedando reducida la cantidad de $\mathrm{SC}_{3}$ formable.

c) $\mathrm{Si}$ el $\mathrm{SC}_{2}$ que contiene el clínker es insuficiente para equilibrar el $\mathrm{K}_{2} \mathrm{O}$ presente, se descompondrá el $\mathrm{SC}_{3}$ dando lugar a cal libre. 
En la revisión llevada a cabo por Newkirk, en 1952, sobre el cálculo de las fases del clínker presenta un procedimiento para calcular aproximadamente la composición potencial del clínker, incluyendo los compuestos antes mencionados (7).

De las reacciones A y B podemos deducir la siguiente fórmula, calculada estequiométricamente:

$$
\mathrm{X}=\mathrm{K}_{2} \mathrm{O}_{\mathrm{ck}}-\left(\mathrm{SO}_{3 \mathrm{ck}} \times 0,86+\mathrm{SC}_{2 \mathrm{ck}} \times 0,034\right)
$$

Siendo:

$\mathrm{X}=\% \mathrm{~K}_{2} \mathrm{O}$ libre para combinarse con $\mathrm{SC}_{3}$ dando lugar a la reacción $\mathrm{A}$.

$\mathrm{K}_{2} \mathrm{O}_{c k}=$ contenido de $\mathrm{K}_{2} \mathrm{O}$ en el clínker.

$\mathrm{SC}_{2 \mathrm{ck}}=$ contenido de bicálcico en el clínker.

$\mathrm{SC}_{2}=$ contenido de bicálcico en el clínker.

Se puede considerar como valor aceptable de $\mathrm{K}_{2} \mathrm{O}$ libre, sin que se produzcan valores demasiado altos de cal libre un $0,3 \%$, según figura 6 (17).

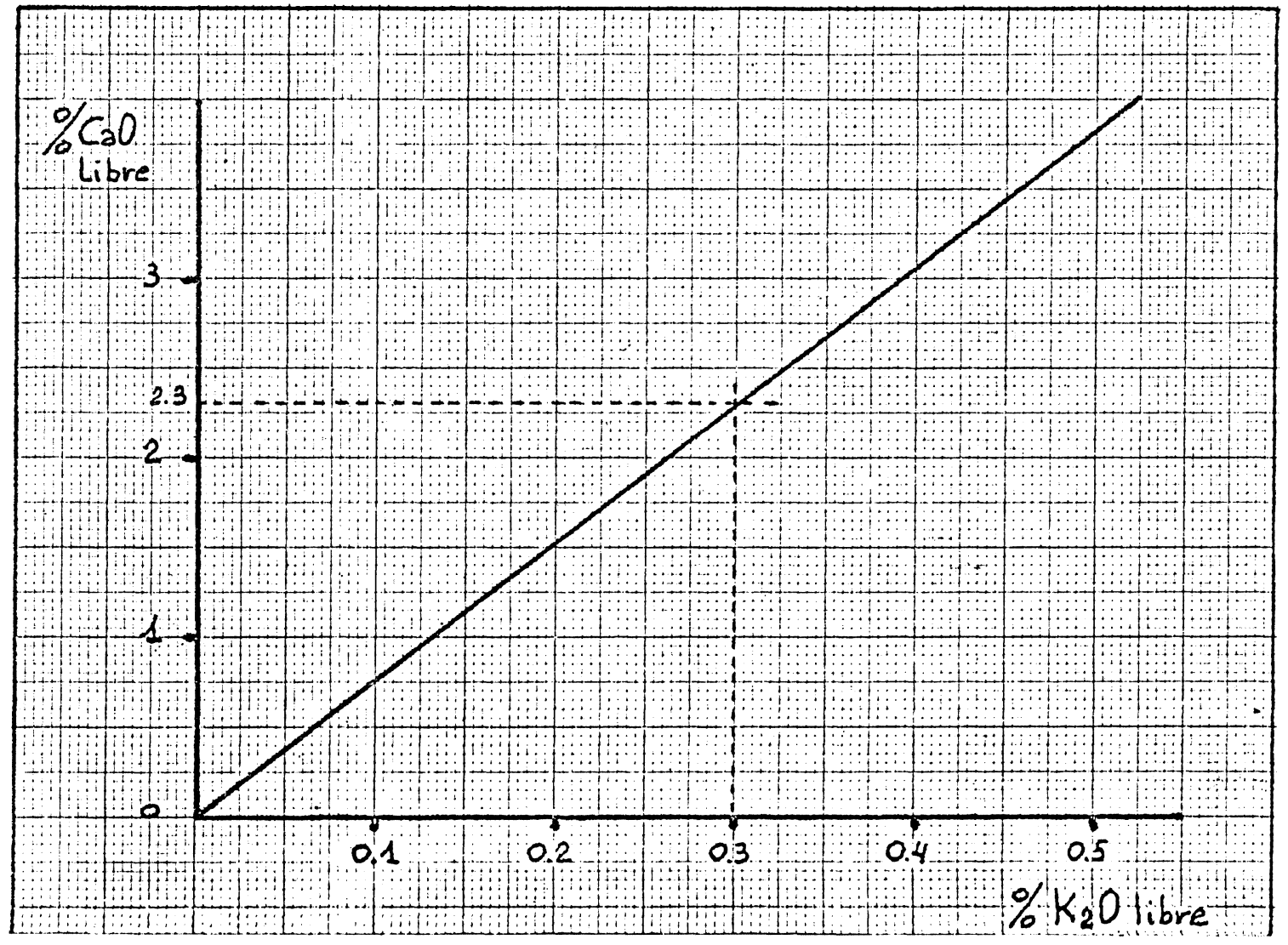

Fig. 6

\subsubsection{Aplicación al caso de utilizar fuel-oil}

Utilizando fuel-oil como combustible, tenemos en el clínker valores de 0,6-0,7 \% de $\mathrm{SO}_{3}$. 
Aplicando el balance $\mathrm{SO}_{3}$-ácalis en el caso de nuestro crudo, se llega a la conclusión de que con valores inferiores a $1,5 \%$ de $\mathrm{K}_{2} \mathrm{O}$ en el clínker no deben aparecer problemas. Como que efectivamente el contenido de $\mathrm{K}_{2} \mathrm{O}$ en el clínker es inferior a $1,5 \%$, no tenemos problemas en el caso de utilizar fuel-oil como combustible.

\subsubsection{Aplicación al caso de utilizar mezcla de gas-fuel}

Utilizando una mezcla de combustibles (17 \%en calorías de fuel), tenemos en el clínker valores de 0,3-0,4 de $\mathrm{SO}_{3}$.

Realizando el balance en este caso, nos encontramos en el límite aceptable. Ello concuerda con los valores de cal libre inferiores a 2 obtenidos en el primer período de utilización de mezcla de combustibles.

Veamos lo que ocurrió en el período del año 71 cuando notamos de nuevo las anomalías en el clínker, y que incluso llegamos a aumentar el porcentaje de fuel en el combustible empleado.

Analizando los datos de análisis de álcalis en el período 1970-71, en el que se habían realizado los ensayos de mezclas de combustibles, apreciamos un aumento considerable de $\mathrm{K}_{2} \mathrm{O}$ en una de nuestras materias primas, precisamente en el período en que notamos el cambio de características del clínker, usando la misma mezcla de combustible (fig. 7).

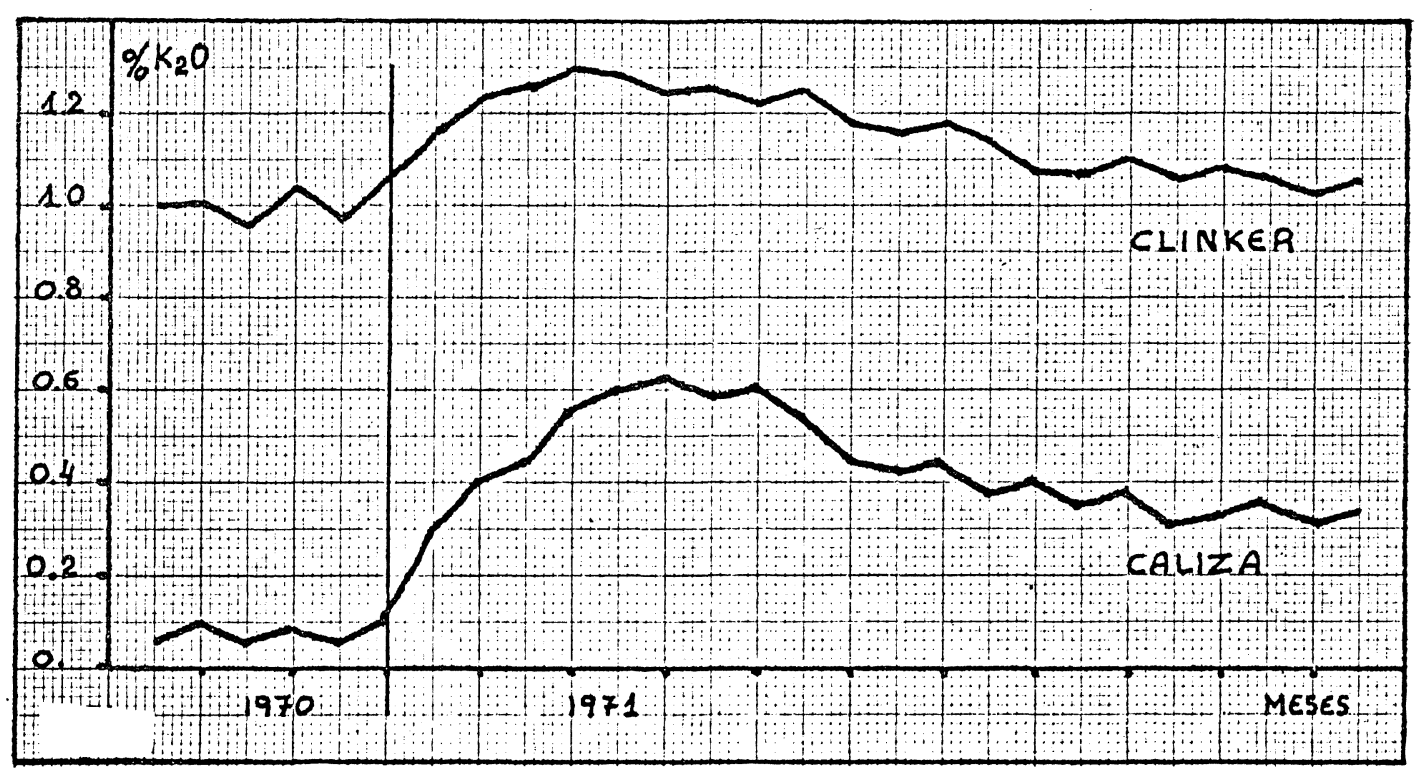

Fig. 7

Ello afectó al contenido de $\mathrm{K}_{2} \mathrm{O}$ en el clínker, quedando el balance $\mathrm{SO}_{3}$-álcalis completamente desplazado en el sentido de provocar la aparición de cal libre en cantidad considerable.

\subsubsection{Aplicación al caso de utilizar gas natural}

En el caso de utilizar como combustible gas natural, obtenemos en el clínker valores de $\mathrm{SO}_{3}$ inferiores al $0,2 \%$, por lo que nos quedan mayores porcentajes de $\mathrm{K}_{2} \mathrm{O}$ libre, con sus correspondientes consecuencias. 
En la figura 8 se representan los valores de cal libre que obtuvimos, tanto en el caso de utilizar fuel-oil, como gas natural y mezcla de ambos.

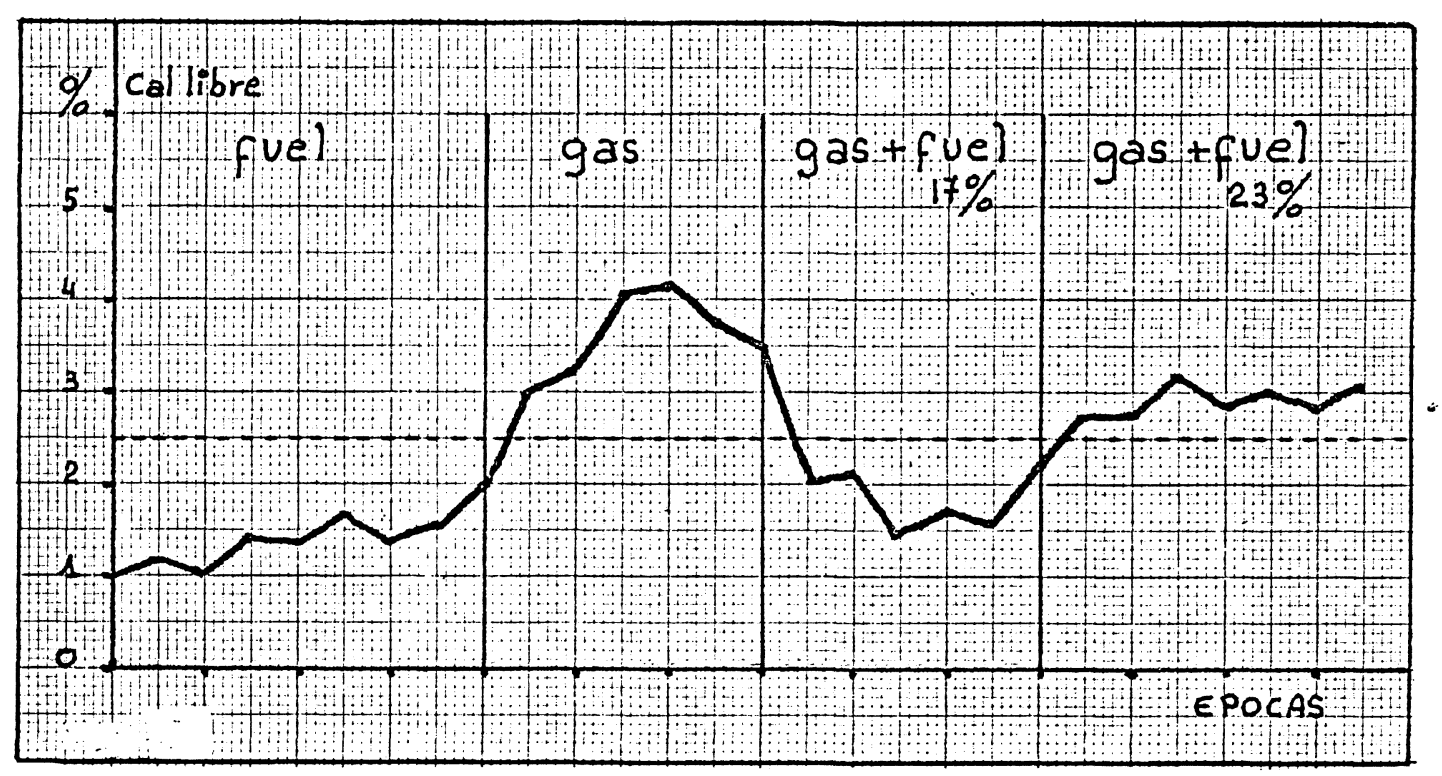

F.g. 8

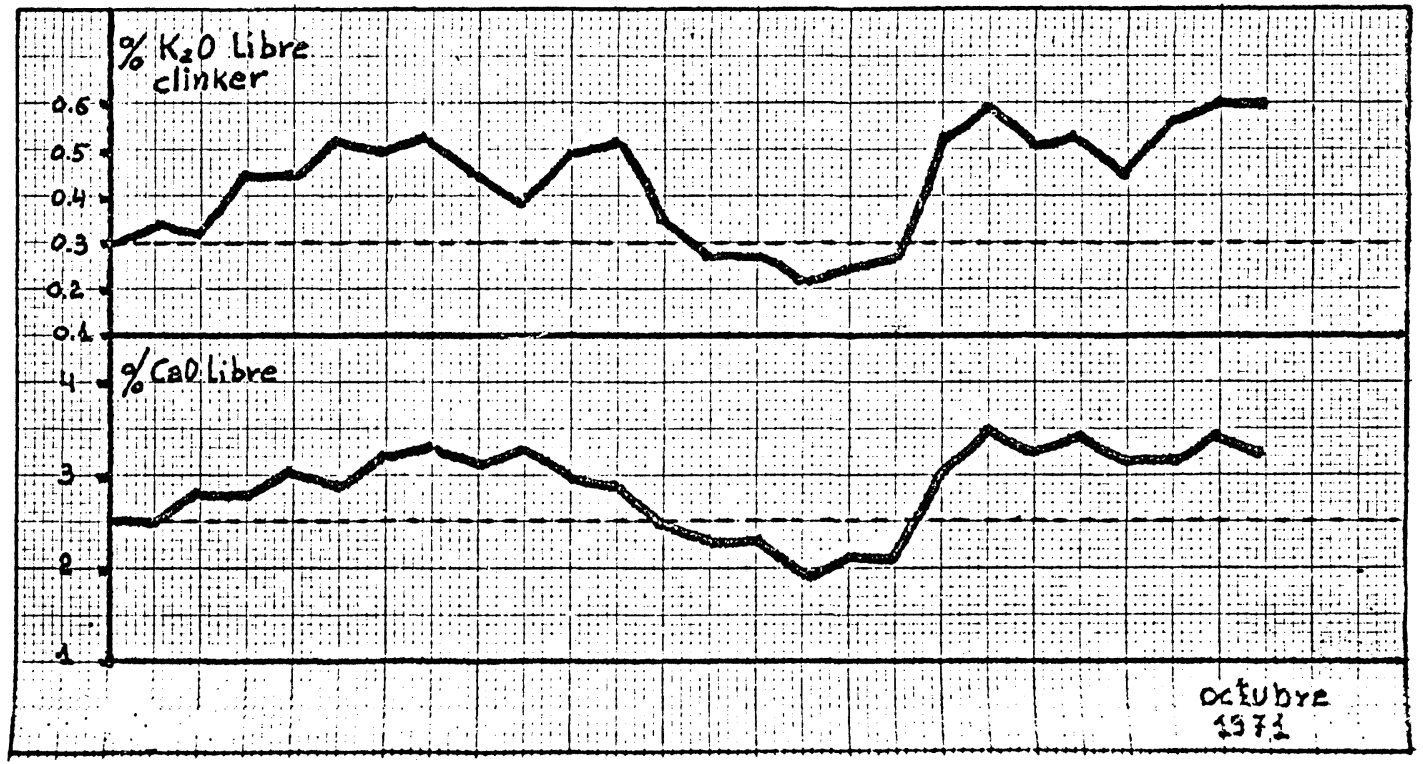

Fig. 9

También es interesante la figura 9 en la que representamos valores de cal libre y de $\mathrm{K}_{2} \mathrm{O}$ libre, calculado según la fórmula vista anteriormente y en la cual se ve una clara concordancia. 


\subsection{Solución adoptada}

A la vista de lo expuesto anteriormente, llegamos a la conclusión de que el problema estaba en la falta de S para poder combinar los álcalis de nuestro crudo.

Aprovechando las características de nuestro horno Humboldt, que por su tamaño ha demostrado ser un excelente horno-piloto, en el que es posible realizar pruebas sin exponer la calidad global del clínker fabricado por los otros hornos, pensamos que una dosificación adecuada de $\mathrm{SO}_{4} \mathrm{Ca}$ en el crudo podría solucionar el problema.

Se llevó a cabo la prueba dosificando el $\mathrm{SO}_{4} \mathrm{Ca}$ directamente al horno, junto con la alimentación, y los resultados fueron francamente espectaculares. Varias pruebas de corta duración bastaron para darnos cuenta de que se resolvía completamente el problema. Los efectos se notaron a las pocas horas de iniciarse la prueba (fig. 10), desapareciendo más lentamente al interrumpirla.

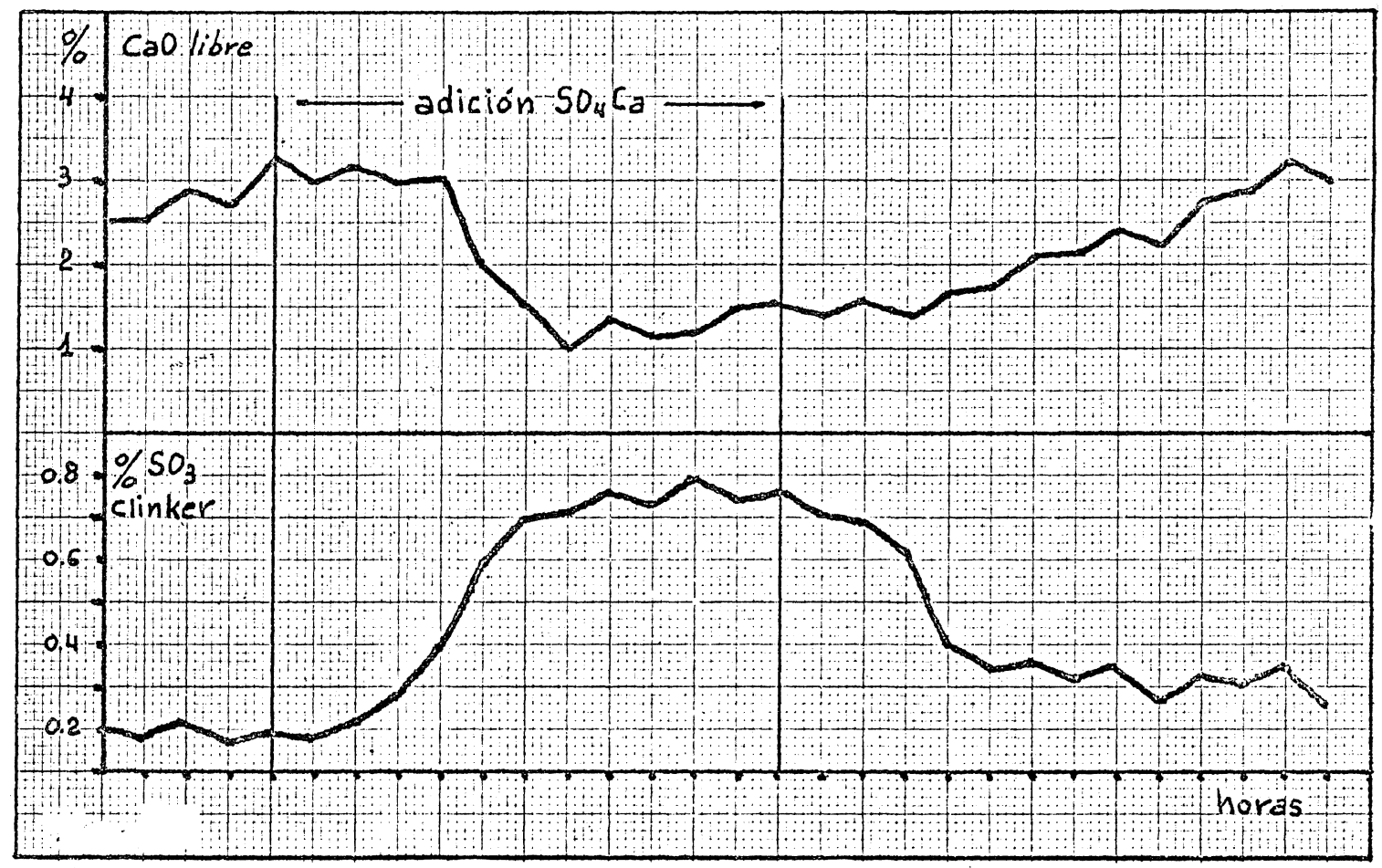

Fig. 10

La siguiente fase fue plantear la adición de $\mathrm{SO}_{4} \mathrm{Ca}$ como solución definitiva, pensando ya en volver a emplear gas natural en el horno Smidth de 1.100 t/día.

Una vez resuelto el problema de la dosificación de $\mathrm{SO}_{4} \mathrm{Ca}$ en el crudo, empezamos nuevamente a utilizar gas natural en este horno, con resultados excelentes y que siguen en la actualidad.

Dado que actualmente disponemos de otro horno Smidth, 4 etapas, para $1.500 \mathrm{t} /$ día, en el que se emplea como combustible el fuel-oil, diariamente se comparan los clínkeres obtenidos con ambos combustibles, siendo las calidades iguales en todos los aspectos.

Actualmente estamos efectuando la instalación de gas natural en este horno último con el fin de tener también la posibilidad de utilizar en él este combustible. 


\section{CONCLUSIONES}

Las variaciones que hemos observado en rendimientos, comparando ambos combustibles, son inferiores a las que normalmente tenemos en un período largo con un mismo combustible, ya sea gas o fuel, con lo cual, en nuestro caso particular, es difícil precisar cuál de los dos es más económico.

Lo que sí se puede asegurar es que hemos encontrado el empleo del gas natural más limpio, más cómodo y con instalaciones complementarias más sencillas y reducidas; todo ello se puede aplicar especialmente durante los períodos de arranque. El cambio de combustible no ha ocasionado ningún trastorno grave en la adaptación de los operarios horneros para su uso.

Sin embargo cabe señalar que, siendo un combustible gaseoso, se deben mantener adecuadamente las condiciones de seguridad, especialmente en lo que se refiere a las operaciones de manipulación y trabajo (purgar completamente el horno de humos, gases o aire antes del encendido, vigilancia de las posibles fugas en la red, control periódico de las válvulas de seguridad y respeto a las normas de seguridad durante las operaciones de mantenimiento o reformas en la red).

En general el empleo de gas natural como sustituto del fuel-oil será más o menos interesante dependiendo de:

- La situación geográfica, que condicionará su disponibilidad.

- Los tipos de hornos, que harán considerar relativamente en mayor o menor grado las modificaciones de consumo o producción.

- Las características de las materias primas, que condicionarán que el período de adaptación sea más o menos largo y difícil.

\section{REFERENCIAS}

(1) E. C. Goldman: The impact of technological developments on future prospects for natural gas. 13 th world gas conference. London 1976.

(2) Nations Unies: L'utilisation du Gaz l'Industrie du ciment. 1976.

(3) C. H. PURkIs: The utilisation of gas a global review 13 World gas conference. London 76 . IGU/ /T3-76.

(4) Coloquio Internacional de marqueting gasista 1976.

(5) P. Palomar: Panorama de la industria del cemento europea. Cemento y Hormigón. XI-1975.

(6) Frederik M. LeA: The Chemistry of Ciment and Concrete. Edward Arnold (publishers) 1td. 1970. Págs. 73, 104, 142, 154

(7) R. H. Bogue: The Chemistry of Portland Cement. Reinhold Publishing Corporation. New York 1975. Págs. 429, 452, 459.

(8) H. F. W. TAYloR: La Química de los cementos. Enciclopedia de la química Industrial. Vol II. Ediciones Urmo. Bilbaa 1967. Págs. 109, 115, 111, 116.

(9) Fritz KeIl: Cemento. Edt. Técnicos Asociados. Barcelona 1973. Págs. 66, 391, 400.

(10) J. Garstka, W. JANNSEN : Le rendement d'utilisation du gaz naturel dans les cimentéries. 13th world gas conference. London 1976. IGU/F8-76.

(11) Walter H. Duda: Cemnet Data Book. Bauverleg GmbH 1976.

(12) A. Devillers, R. Leduc: L'utilisation du Gaz Naturel dans l'industrie du ciment. Association technique de l'industrie du gaz en France. Congrès 1970.

(13) D. MARQUE: L'utilisation du gaz en cimentérie. Gas warme international. Nov. 1971.

(14) G. Datschefski: Natural gas in the cement Manufacture. Gas warme international. Nov. 1971.

(15) R. LEDUC: L' utiilsation du gaz naturel dans les industries de fabrication des ciments et chaux. 1972.

(16) A. Gerard: Contribution a l'étude d'un bruleur pour gaz a difusion pour le chauffage des fours rotatifs de grand capacité. Revue Générale du Gaz. 3-4-73.

(17) J. M. Fernandez Paris: Microscopía del clínker de cemento Portland. I.E.T.c.c. 1968. Pág. 45. 